\title{
Magnetohydrodynamic equilibrium of plasma ball lightning
}

\author{
Hanming Wu and Yunming Chen \\ Institute of Mechanics, Chinese Academy of Sciences, Beijing 100080, People's Republic of China
}

(Received 29 November 1988; accepted 28 March 1989)

\begin{abstract}
A study is done of magnetohydrodynamic (MHD) equilibrium of a self-confined plasma ball imbedded in an atmosphere with constant pressure $p_{0}$. It is found that there is more than one equilibrium state for the plasma ball and the average pressure inside the ball is less than $p_{0}$. It is also shown that the force-free magnetic configuration cannot exist in the equilibrium state. In comparison with a spheromak, the magnetic axis in ball lightning drifts toward the center about $10 \%$.
\end{abstract}

\section{INTRODUCTION}

Ball lightning is a very interesting problem and has puzzled many scientists for more than two centuries. There are two main problems: First, how can a plasma ball be stably confined in a certain limited volume for a long time without any auxiliary magnetic field? Second, where does the energy of ball lightning come from? In the present paper, the answer to the former question is partly given and some theoretical results are discussed qualitatively.

Among the wide variety of possible electric and magnetic structures that may occur in ball lightning, a self-confinement configuration seems most reasonable. A large body of literature on self-confinement problems has been developed and can be found in a number of general reviews on solar physics. ${ }^{1-3}$ Meanwhile, plasma balls with spherical configurations have received wide attention in laboratories (e.g., spheromaks and rotamaks-the so-called compact torus). ${ }^{4-6}$ Nevertheless, there are few references that deal with a spherical plasma ball with self-confinement to explain how a plasma ball can be in magnetohydrodynamic (MHD) equilibrium as well as be stable against MHD perturbations. ${ }^{7,8}$

In the present paper, after an analysis of the equilibrium condition by means of the Virial theorem, the solution of the Grad-Shafranov equation derived from $\nabla p=\mathbf{J} \times \mathbf{B}$ is obtained. We then present the magnetic configuration as well as the pressure distribution of the plasma ball.

\section{THE GENERAL MHD EQUILIBRIUM CONDITION}

Consider plasma ball lightning carrying a toroidal current density $J_{\phi}$ and a poloidal current density $J_{p}$. The plasma ball is imbedded in an atmosphere of constant pressure $p_{0}$. The gravitation is neglected because the gravitational force is much less than the Lorentz force. The MHD equilibrium can then be described by the following equation:

$$
\boldsymbol{\nabla} p=\mathbf{J} \times \mathbf{B}, \quad \nabla \times \mathbf{B}=\mu_{0} \mathbf{J}, \quad \nabla \cdot \mathbf{B}=\mathbf{0} .
$$

On the spherical boundary surface, $p=p_{0}, \mathrm{~B}=0,\left(r=r_{0}\right)$, where $\mu_{0}, p, \mathbf{J}$, and $\mathbf{B}$ are the magnetic permeability, pressure, current density, and magnetic induction, respectively, and $r_{0}$ is the radius of the plasma ball.

To avoid solving Eq. (1) directly, we can discuss some MHD equilibrium properties qualitatively by means of the Virial theorem first. It is well known that the Virial theorem implies that all equilibrium plasma systems must satisfy the following relation:

$$
\int_{\tau} \frac{3 p+B^{2}}{2 \mu_{0}} d \tau=\int_{\Sigma}\left(\frac{2 p+B^{2}}{2 \mu_{0}} \mathbf{r} \cdot d \mathbf{s}-(\mathbf{B} \cdot \mathbf{r})\left(\frac{\mathbf{B} \cdot d \mathbf{s}}{\mu_{0}}\right)\right) .
$$

This relation implies that a finite plasma volume can be in equilibrium only when there are some fixed conductors carrying current that generate a magnetic field to balance the plasma pressure. That is why in all laboratory plasma equilibrium configurations an auxiliary magnetic field has to be used to keep the plasma in equilibrium.

Attention must be paid to the fact that the Virial theorem has an important premise, namely, that the ambient presssure is assumed to be zero.

For example, in a tokamak plasma, because of the curvature of the loop, both the Lorentz force and plasma pressure give rise to nonzero net contributions to the force in the major radial direction. The total major radial force can be explicitly calculated as

$$
F=\frac{\mu_{0} I_{p}^{2}}{2}\left(\ln \frac{8 R}{R_{a}}+\frac{l i}{2}-1\right)+2 \pi^{2} R_{\alpha}^{2}\left(\langle p\rangle-\frac{\left\langle B_{\phi}^{2}\right\rangle}{2 \mu_{0}}\right),
$$

where $l i$ is the internal inductance per unit length; $\langle p\rangle$ and $\left\langle B_{\phi}^{2}\right\rangle$ are the internal average of $p$ and $B_{\phi}^{2}$, respectively; $I_{p}, R_{a}$, and $R$ are the currents in the poloidal direction, minor radius, and major radius, respectively. It is obvious that only when $\langle p\rangle\langle 0$ can $F$ be zero. Therefore, it is impossible for a plasma in a tokamak to be in equilibrium without an appropriate auxiliary magnetic field, which is usually called a vertical field. This conclusion coincides with that of the Virial theorem.

\section{DETAILED MAGNETIC STRUCTURE}

Assume the equilibrium plasma ball to be axially symmetric, i.e., $\partial / \partial \phi=0$. The equilibrium magnetic flux function $\psi$ should then satisfy the Grad-Shafranov equation in the form of spherical coordinates $(r, \theta, \phi)$ as follows:

$$
\widehat{L} \psi=-2 \mu_{0} \pi r \sin \theta \cdot J_{\phi},
$$

where

$$
\begin{aligned}
& \hat{L}=\frac{\partial^{2}}{\partial r^{2}}+\frac{\partial^{2}}{r^{2} \partial \theta^{2}}-\frac{\cos \theta}{r^{2} \sin \theta} \frac{\partial}{\partial \theta}, \\
& J_{\phi}=2 \pi r \sin \theta \frac{d p}{d \psi}+\frac{\mu_{0}}{4 \pi r \sin \theta} \frac{d I^{2}}{d \psi} .
\end{aligned}
$$

Here, both $p$ and $I$ are functionals of $\psi$ only. 
as

The corresponding boundary condition can be rewritten

$$
\left.\psi\right|_{r_{\mathrm{o}}}=0,\left.\quad \hat{r} \nabla \psi\right|_{r_{\mathrm{o}}}=0,
$$

which means that the ambient pressure $p_{0}$ is a constant and $B=0$ as well as $\nabla p=0$ on the boundary surface.

The Virial theorem implies that a force-free field $(\mathrm{J} /$ ) B) may exist in such a region, in which the ambient pressure $p_{0}$ should be greater than the average $\langle p\rangle$ inside the region. Therefore, it seems reasonable to consider the force-free field first.

Let the equilibrium magnetic field be

$$
\boldsymbol{\nabla} \times \mathbf{B}=k \mathbf{B},
$$

where $k$ is a constant called the force-free factor. ${ }^{9}$ Then $p$ and $I$ in Eq. (3) become

$$
p(\psi)=\mathrm{constant}, \quad I(\psi)=k \psi / \mu_{0},
$$

i.e., $J_{\phi}=k^{2} \psi /\left(2 \pi \mu_{0} r \sin \theta\right)$.

Equation ( 3 ) can be rewritten as

$$
\hat{L} \psi=-k^{2} \psi \text {. }
$$

Obviously, the complementary solution of the equation is

$$
\psi=a_{l} r \sin \theta \cdot P_{l}^{1}(\cos \theta) j_{l}\left(k_{l} r\right),
$$

where $P_{l}^{1}(\cos \theta)$ is the associated Legendre function, $j_{l}(k r)$ is the spherical Bessel function of order $l$. From the boundary condition (4), we find that $a_{l}=0$, i.e., there is only a trivial solution for the force-free field configuration. This implies that the force-free field can not exist in plasma ball lightning.

Now, consider another model in which the pressure and current are

$$
p(\psi)=a \psi+p_{0}, \quad I(\psi)=c \psi
$$

where $a$ and $c$ are constants. The toroidal current density is then

$$
J_{\phi}=2 \pi a_{0} r \sin \theta+\left(\mu_{0} c_{0}^{2} / 4 \pi r \sin \theta\right) \psi,
$$

and Eq. (3) can be expressed as

$$
\widehat{L} \psi=-a r^{2} \sin ^{2} \theta-b^{2} \psi,
$$

where $a=4 \pi^{2} \mu_{0} a_{0}, b=\mu_{0} c_{0}$. We manage to find the complementary solution of Eq. (10) as follows:

$$
\psi=r \sin ^{2} \theta\left[\alpha j_{1}(b r)-a r / b^{2}\right] \text {. }
$$

The constant $\alpha$ can be determined from the boundary condition (4) as

$$
\alpha=a r_{0} / b^{2} j_{1}\left(b r_{0}\right) \text {, }
$$

where $b$ is the root of $j_{2}\left(b r_{0}\right)=0$. It is obvious that $b=\left\{b_{n}\right\}$ is an infinite ensemble since $j_{2}(x)$ is a periodic function. The corresponding solution of $b_{n}$ is

$$
\psi_{n}=\frac{a r \sin ^{2} \theta}{b_{n}^{2}}\left(\frac{r_{0} j_{1}\left(b_{n} r\right)}{j_{1}\left(b_{n} r_{0}\right)}-r\right) .
$$

This is the particular solution of Eq. (10) with the boundary condition (4). Because the equation dealt with in this paper is an eigenvalue one, the equilibrium state is obviously nonunique.

The position of the magnetic axis $r_{m}$ can be obtained by

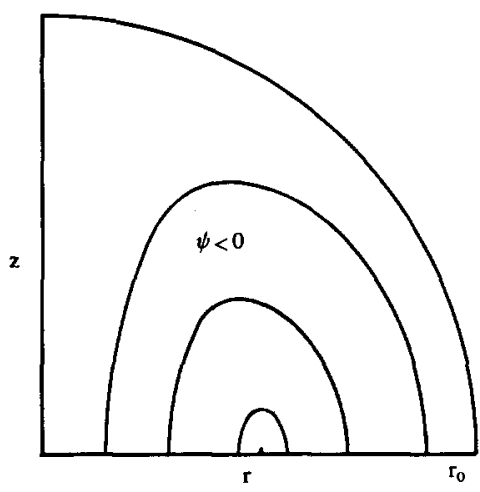

FIG. 1. Magnetic flux function $\psi, a=1, b_{1} r_{0}=5.76346$.

means of $\nabla \psi=0$, i.e.,

$$
\begin{aligned}
2 r_{m}^{3} j_{1}\left(b_{n} r_{0}\right) b_{n}^{2} / r_{0}= & b_{n} r_{m} \cos \left(b_{n} r_{m}\right)+\left(b_{n}^{2} r_{m}^{2}-1\right) \\
& \times \sin \left(b_{n} r_{m}\right)=0,
\end{aligned}
$$

where $\theta=\pi / 2$. Using the solution (13), we can obtain the magnetic field $\mathrm{B}_{n}=\left(B_{n r}, B_{n \theta}, B_{n \phi}\right)$, where

$$
\begin{aligned}
& B_{n r}=\frac{2 a \cos \theta}{b_{n}^{2} r}\left(\frac{r_{0} j_{1}\left(b_{n} r\right)}{j_{1}\left(b_{n} r_{0}\right)}-r\right), \\
& B_{n \theta}=\frac{a \sin \theta}{r b_{n}^{2}}\left[2 r-\frac{r_{0}}{j_{1}\left(b_{n} r_{0}\right)}\left(j_{1}\left(b_{n} r\right)+r \frac{d}{d r} j_{1}\left(b_{n} r\right)\right)\right], \\
& B_{n \phi}=\frac{a \sin \theta}{2 \pi b_{n}}\left(\frac{r_{0} j_{1}\left(b_{n} r\right)}{j_{1}\left(b_{n} r_{0}\right)}-r\right) .
\end{aligned}
$$

For two equilibrium states corresponding to $b_{1}$ and $b_{2}$, the magnetic flux functions $\psi$ are displayed clearly in Figs. 1 and 2, where $b_{1} r_{0}=5.763459, b_{2} r_{0}=9.095$. The pressure distribution is shown in Fig. 3, which proves that the average pressure inside the plasma ball is less than the ambient pressure $p_{0}$.

\section{COMPARISON BETWEEN BALL LIGHTNING AND A SPHEROMAK}

Consider the equilibrium state of $n=1$, i.e., there is only one magnetic axis in the plasma ball. Assume that the radius $r_{0}$ and the maximum magnetic flux $\psi_{m}$ in both the ball lightning and the spheromak are the same.

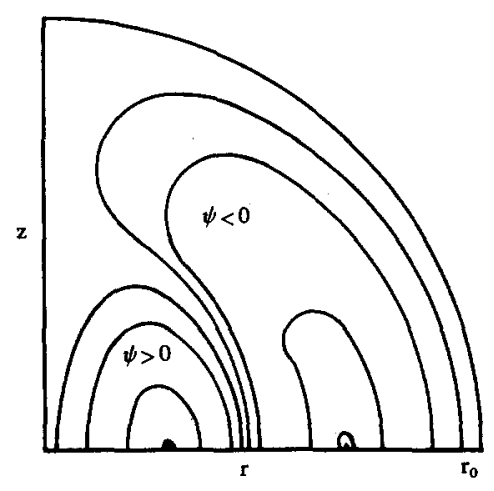

FIG. 2. Magnetic flux function $\psi, a=1, b_{2} r_{0}=9.095$. 


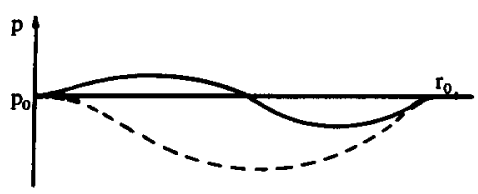

FIG. 3. Pressure distribution inside the plasma ball: $\ldots b_{1} r_{0}=5.76346,-b_{2}$ $r_{0}=9.095$.

Figure 1 shows that the axis lies at $0.5 r_{0}$ as compared with the magnetic axis in a spheromak, which lies at $0.6 r_{0}$. The reason why the magnetic axis in ball lightning drifts toward the center is that without any auxiliary magnetic field the weaker magnetic pressure inside the ball results in a smaller magnetic axis circle.

From Refs. 4-6, the safety factors $q=d \psi_{\phi} / d \psi_{p}$ are $q_{A}=0.825$ and $q_{\Gamma}=0.72$ at the magnetic axis and the boundary surface, respectively. The $q_{A}$ and $q_{\Gamma}$ in the present paper can be easily obtained from (15)

$$
\begin{aligned}
& q_{A}=\left.\lim _{\epsilon \rightarrow 0} \frac{\epsilon B_{\phi}\left(r_{m}\right)}{r_{m} B_{\theta}\left(r_{m}+\epsilon\right)}\right|_{\theta=\pi / 2}=-0.04578, \\
& q_{\Gamma}=b r_{m} / 4 \pi=0.23531 .
\end{aligned}
$$

Now, the estimation of the magnetic shear $v$ is

$$
v=\left|\frac{d q}{d \psi} q^{-1}\right| \sim\left\{\begin{array}{cc}
2.775 & \text { (spheromak), } \\
32.97 & \text { (ball lightning). }
\end{array}\right.
$$

\section{CONCLUSION}

We have discussed the MHD equilibrium properties for ball lightning. The following physical conclusions are arrived at: (1) The force-free field cannot exist in the equilibrium state. (2) The pressure distribution inside the plasma ball, from Fig. 3, shows that the average pressure inside the plasma ball is less than the ambient one, namely, the pressure gradient directs outside.

\section{ACKNOWLEDGMENTS}

The authors are grateful to Yue Su for beneficial discussions. Special thanks should be expressed to Professor Minglun Xue for his encouragement and concern.

'J. C. Brown and D. F. Smith, Rep. Prog. Phys. 43, 125 (1980).

${ }^{2} Z$. Svestk, Solar Flares (Reidel, Dordrecht, The Netherlands, 1976).

${ }^{3}$ M. L. Xue and J. Chen, Sol. Phys. 84, 119 (1983).

${ }^{4}$ M. N. Rosenbluth and M. N. Bussac, Nucl. Fusion 19, 489 (1979).

${ }^{5}$ W. N. Hugrass, I. R. Jones, and M. G. Phillips, Nucl. Fusion 19, 1546 (1979).

${ }^{6} \mathrm{H}$. Wu and L. Pan, Acta Phys. Sin. 33, 1100 (1984).

${ }^{7}$ B. M. Smirnov, Phys. Rep. 152, 178 (1987).

${ }^{8}$ Y. Zou, Adv. Atmos. Sci. 6, 1 (1989).

${ }^{9}$ L. Pan, Acta Astron. Sin. 20, 182 (1979). 